\title{
Future breakthroughs in the genetics of pulmonary arterial hypertension
}

Pulm Circ 2013;3(3):451-453. DOI: 10.1086/674305.

Recent years have witnessed a number of new discoveries relating to underlying genetic influences in patients with idiopathic and heritable pulmonary arterial hypertension (PAH). It is now 13 years since the identification of mutations in the gene encoding the bone morphogenetic protein type II receptor (BMPR2) as the major inherited cause of PAH, ${ }^{1,2}$ based on classical linkage analysis in affected pedigrees and sequencing of genes in the candidate region. Mutations in this gene account for approximately $70 \%$ of heritable and $16 \%-25 \%$ of idiopathic $\mathrm{PAH}^{3}$ In the intervening years, investigators have begun to unravel how mutations in this gene cause disease. The reward is that therapeutic approaches targeting this pathway are now being realized, at least in preclinical models. ${ }^{4-6}$

Further mutations have been identified based on candidate gene approaches in this same pathway: for example, mutations in the activin-like kinase 1 receptor (ALK1) and the transforming growth factor $\beta$ (TGF- $\beta$ ) accessory receptor, endoglin. ${ }^{7,8}$ The fact that BMPR2 interacts directly with ALK1 on endothelial cells to signal in response to the ligands BMP9 and $10^{9}$ is likely telling us that the endothelial cell is a major cell type, at least in the initiation of this disease. The identification of rare mutations in Smad1, Smad4, and Smad8 strongly implicates this canonical signaling pathway downstream of the BMP receptors as important. Thus genetic discoveries can accelerate our understanding of disease pathobiology or add strong support to existing hypotheses.

Of course, there are likely to be many mechanisms by which pulmonary hypertension can develop, without involving the BMP pathways. Recent identification of mutations in caveolin-1, the potassium chan- nel KCNK $3,{ }^{10}$ and possibly TBX4, point toward other mechanisms of disease, although possible links with the BMP pathway remain to be explored for most of these. Each new discovery offers a potential therapeutic intervention that targets a common cellular process in PAH as well as providing new insights into mechanisms. It does not matter that a mutation might only affect $1 \%$ of PAH patients. This does not diminish the potential importance of the pathway identified, which may have broad implications for therapeutic approaches in other forms of PAH. It may also point toward a unifying cellular mechanism of disease. Hence the pressing need to harness the rapidly advancing technical and bioinformatic developments in disease gene discovery that are transforming clinical medicine, to complete our understanding of the entire genetic architecture of PAH. In this way we should be able to view the various pathways implicated, make connections between them, and identify the "sweet spots" for therapeutic intervention.

Much work remains to be done. Discoveries will depend on the application of next-generation sequencing technology in rare but informative families with disease in which known mutations have been excluded, as well as large-scale analysis for rare genetic variation in large cohorts of patients with idiopathic or heritable PAH. One approach yielding new findings ${ }^{10}$ is the use of exome sequencing: sequencing the $2 \%$ or so of the genome that actually encodes for proteins. This technology is cost-effective-about $\$ 1,000$ per exome (compare this with the cost of sequencing the entire genome in $2001-\$ 100$ million). The costs are still falling, and soon it may be possible to sequence the entire genome of an individual for $\$ 1,000$. A major challenge remains in sifting through 
the information generated by DNA sequencing for the relevant mutations. We all carry thousands of genetic variations and tens of rare potentially deleterious mutations in our genomes. Identification of causal mutations requires complex analysis and filtering of candidate variants and, ultimately, proof in tissues, cell, and model organisms. Once we have mined the exome data for information, the next challenge will be to determine the contribution of the regulatory regions encoded in the remainder of the genome.

In addition to these sequencing approaches, genome-wide association studies (GWAS) seek a statistical association between common genetic variants and the presence of disease. This usually requires large numbers of affected individuals compared with a control population. This approach can identify common variants that influence the susceptibility to disease and may help narrow down the hunt for rare variants from sequencing studies. Since, even in an individual with BMPR2 mutations, the risk of developing disease is only $20 \%-30 \%$, there may be common genetic variants that contribute to disease penetrance. Common genetic variation may also indicate the nature of gene-environmental interactions that play important roles in disease manifestation. The only published GWAS in idiopathic PAH to date identified an association with cerebellin- $2,{ }^{11}$ though this study was too small to confirm other potential associations. Ultimately, larger studies and meta-analyses will be required to dissect the small but important contributions of common genetic variants to PAH susceptibility. Again, each of the associated loci may indicate clues to mechanisms and potential targets for therapy as well as providing prognostic information on clinical outcomes and response to therapy.

A complete picture of the genetic basis of PAH and an understanding of the contribution of genetics to clinical management will require the study of large cohorts of patients, which is a challenge for a rare disease. The rarity mandates national and international collaborations, with standardized approaches to phenotyping and sampling. Several of these are now under way (e.g., http://www.clinicaltrials.gov/ct2 /show/NCT01907295). The application of large-scale and cost-effective next-generation sequencing technologies to these cohorts, coupled with advances in bioinformatics and computational biology, are likely to reveal new and exciting insights into the pathobiology of PAH in the next few years. We should also note that careful and systematic clinical phenotyping of PAH will be vital to assist the analysis of sequencing data. We are entering an era where genomic information will find a central place in the diagnosis and management of clinical disease. The next few years may provide the basis for a full molecular genetic classification of PAH that will enhance existing systems of classification of this disease.

Nicholas W. Morrell, Department of Medicine, University of Cambridge School of Clinical Medicine, Addenbrooke's and Papworth Hospitals, Cambridge, United Kingdom

nwm23@cam.ac.uk

\section{REFERENCES}

1. International PPHC, Lane KB, Machado RD, et al. Heterozygous germ-line mutations in BMPR2, encoding a TGFreceptor, cause familial primary pulmonary hypertension. Nat Genet 2000;26:81-84.

2. Deng Z, Morse JH, Slager SL, et al. Familial primary pulmonary hypertension (gene PPH1) is caused by mutations in the bone morphogenetic protein receptor-II gene. Am J Hum Genet 2000;67:737-744.

3. Machado RD, Aldred MA, James V, et al. Mutations of the TGF- $\beta$ type II receptor BMPR2 in pulmonary arterial hypertension. Hum Mutat 2006;27:121-132.

4. Dunmore BJ, Drake KM, Upton PD, Toshner MR, Aldred MA, Morrell NW. The lysosomal inhibitor, chloroquine, increases cell surface BMPR-II levels and restores BMP9 signalling in endothelial cells harbouring BMPR-II mutations. Hum Mol Genet 2013;22:3667-3679.

5. Long L, Yang X, Southwood M, et al. Chloroquine prevents progression of experimental pulmonary hypertension via inhibition of autophagy and lysosomal bone morphogenetic protein type II receptor degradation. Circ Res 2013;112: 1159-1170.

6. Spiekerkoetter E, Tian X, Cai J, et al. FK506 activates BMPR2, rescues endothelial dysfunction, and reverses pulmonary hypertension. J Clin Invest 2013;123:3600-3613.

7. Trembath RC, Thomson JR, Machado RD, et al. Clinical and molecular genetic features of pulmonary hypertension in patients with hereditary hemorrhagic telangiectasia. N Engl J Med 2001;345:325-334.

8. Chaouat A, Coulet F, Favre C, et al. Endoglin germline mutation in a patient with hereditary haemorrhagic telangiecta- 
sia and dexfenfluramine associated pulmonary arterial hypertension. Thorax 2004;59:446-448.

9. David L, Mallet C, Mazerbourg S, Feige JJ, Bailly S. Identification of BMP9 and BMP10 as functional activators of the orphan activin receptor-like kinase 1 (ALK1) in endothelial cells. Blood 2007;109:1953-1961.
10. Ma L, Roman-Campos D, Austin ED, et al. A novel channelopathy in pulmonary arterial hypertension. N Engl J Med 2013;369:351-361.

11. Germain M, Eyries M, Montani D, et al. Genome-wide association analysis identifies a susceptibility locus for pulmonary arterial hypertension. Nat Genet 2013;45:518-521. 